\title{
On Weakly Periodic Gibbs Measures of the Potts Model with a Special External Field on a Cayley Tree
}

\author{
M.M. Rahmatullaev \\ Institut of Mathematics \\ 29 Durmon Yuli Str., 100125, Uzbekistan \\ E-mail: mrahmatullaev@rambler.ru
}

Received March 5, 2015, revised January 27, 2016

\begin{abstract}
In the paper, we study the $q$-state (where $q=3,4,5, \ldots$ ) Potts model with special external field on a Cayley tree of order $k \geq 2$. For antiferromagnetic Potts model with such an external field on the Cayley tree of order $k \geq 6$, the non-uniqueness of weakly periodic (non-periodic) Gibbs measures is proved. The weakly periodic Gibbs measures for the Potts model with zero external field are also studied. It is proved that under some conditions imposed on the parameters of the model there can be not less than $2^{q}-2$ such measures.
\end{abstract}

Key words: Cayley tree, Gibbs measure, Potts model, weakly periodic measure.

Mathematics Subject Classification 2010: 82B26 (primary); 60K35 (secondary).

\section{Introduction}

The Potts model on the Cayley tree and its Gibbs measures were studied in [1][4]. In [5], the ferromagnetic three-state Potts model on the Cayley tree of order two was studied, and it was shown that there exists a critical temperature $T_{c}>0$ such that for $T<T_{c}$ there are at least three translation-invariant Gibbs measures and an uncountable number of non-translation-invariant Gibbs measures. In [6], the results of [5] were generalized to the Potts model with a finite number of states on the Cayley tree of an arbitrary (finite) order.

In [7], for an antiferromagnetic Potts model on the Cayley tree it was shown that the translation-invariant Gibbs measure is unique.

(C) M.M. Rahmatullaev, 2016 
The Potts model with a countable number of states in a non vanishing external field on the Cayley tree was considered in [8]. It was proved that this model has a unique translation-invariant Gibbs measure.

Recently, in [9], there were described all translation-invariant Gibbs measures for the Potts model with zero-external field on the Cayley tree of order $k \geq 2$. It was shown that at sufficiently low temperatures their number is $2^{q}-1$.

In $[10,11]$, a notion of weakly periodic Gibbs measure was introduced, and some Gibbs measures were found for the Ising model.

Weakly periodic ground states and weakly periodic Gibbs measures (coinciding with the translation-invariant ones) for the Potts model were studied in [12].

In [13], the existence of weakly periodic (non- translation-invariant) Gibbs measures of the Potts model was proved.

This paper is devoted to the weakly periodic (non-periodic) Gibbs measures for the Potts model with special external field on the Cayley tree. The paper has the following structure. In Sec. 2, we introduce the main definitions and the background. In Sec. 3, we give the results obtained for weakly periodic Gibbs measures. The proofs of all results are given in Sec. 4 .

\section{Definitions and the Background}

Let $\tau^{k}=(V, L)$ be a uniform Cayley tree, where each vertex has $k+1$ neighbors with $V$ being the set of vertices and $L$, the set of edges.

It is known that $\tau^{k}$ can be represented as $G_{k}$, which is the free product of $k+1$ cyclic groups of the second order (see $[7,14,15]$ ).

Fix an arbitrary element $x_{0} \in V$ and correspond it to the unit element $e$ of the group $G_{k}$. Without loss of generality, we assume that the Cayley tree is a planar graph. Using $a_{1}, \ldots, a_{k+1}$, we numerate the nearest neighbors of the element $e$, moving in the positive direction (see Fig.1). Now we numerate the nearest neighbors of each $a_{i}, i=1, \ldots, k+1$ by $a_{i} a_{j}, j=1, \ldots, k+1$. Since all $a_{i}$ have the common neighbor $e$, we give $a_{i} a_{i}=a_{i}^{2}=e$ to it. Other neighbors are numerated starting from $a_{i} a_{i}$ in the positive direction. We numerate the set of all nearest neighbors of each $a_{i} a_{j}$ by $a_{i} a_{j} a_{q}, q=1, \ldots, k+1$ starting from $a_{i} a_{j} a_{j}=a_{i}$ in the positive direction. Iterating this argument, one gets a one-toone correspondence between the set of vertices $V$ of the Cayley tree $\tau^{k}$ and the group $G_{k}$.

The group representation given above is called the right representation, since in this case if $x$ and $y$ are the nearest neighbors on the tree and $g, h$ are the corresponding elements of the group $G_{k}$, then $g=h a_{i}$ or $h=g a_{j}$ for some $i$ or $j$. Similarly, one can define the left representation. 


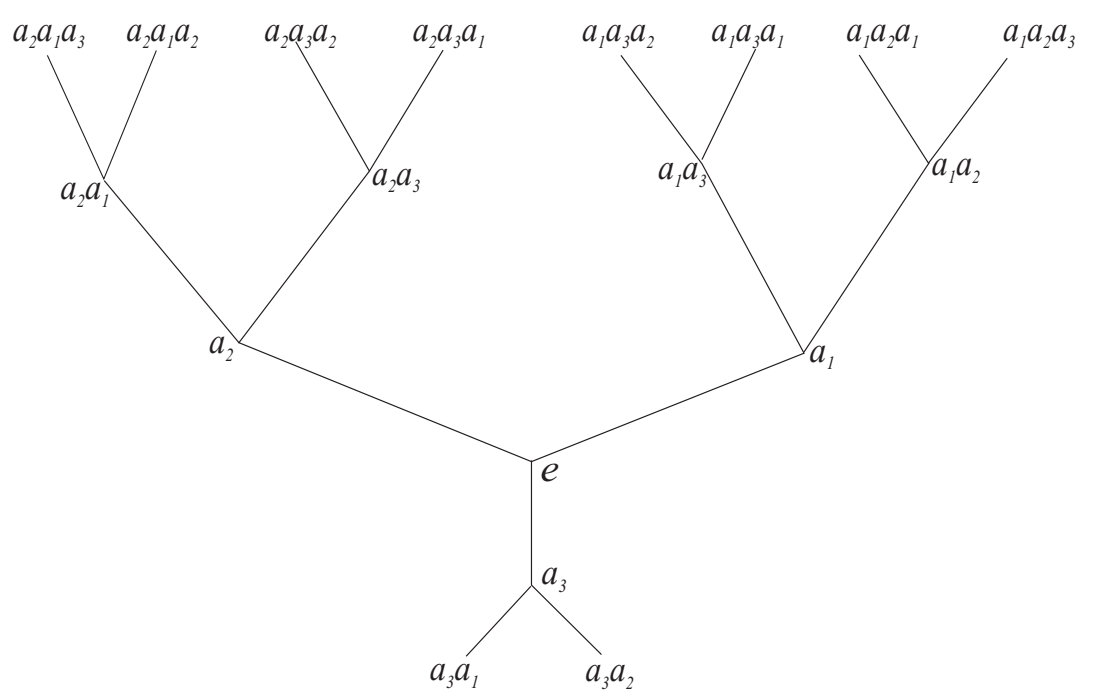

Fig. 1. Some elements of the group $G_{2}$ on the Cayley tree of order two.

In the group $G_{k}$, let us consider the left (right) shift transformations defined as follows. For $g \in G_{k}$, let us set

$$
T_{g}(h)=g h,\left(T_{g}(h)=h g\right), \quad \forall h \in G_{k} .
$$

The set of all left (right) shifts in $G_{k}$ is isomorphic to the group $G_{k}$. Any transformation $S$ of the group $G_{k}$ induces the transformation $\hat{S}$ of the set of vertices $V$ of the Cayley tree.

Therefore, we identify $V$ and $G_{k}$.

Note that the set $\left\{T_{g}: g \in G_{k}\right\}$ is a group with respect to the operation $T_{g} T_{h}=T_{g h}$, and it is called a group of transformations.

Theorem 1. The group of the left (right) shifts on the right (left) representations of the Cayley tree is the group of transformations of the Cayley tree (see $[7,15])$.

For an arbitrary point $x^{0} \in V$, we set $W_{n}=\left\{x \in V \mid d\left(x^{0}, x\right)=n\right\}, V_{n}=$ $\bigcup_{m=0}^{n} W_{m}, L_{n}=\left\{<x, y>\in L \mid x, y \in V_{n}\right\}$, where $d(x, y)$ is the distance between $m=0$
the vertices $x$ and $y$ in the Cayley tree, i.e., the number of edges in the shortest path joining the vertices $x$ and $y$.

We consider the model where the spin variables take values in the set $\Phi=$ $\{1,2, \ldots, q\}, q \geq 3$, and are placed at the vertices of the tree. Then a configuration $\sigma$ on $V$ is defined as a function $x \in V \rightarrow \sigma(x) \in \Phi$. The set of all configurations coincides with $\Omega=\Phi^{V}$. 
The Potts model with external fields is defined by the Hamiltonian

$$
H(\sigma)=-J \sum_{\langle x, y\rangle \in L} \delta_{\sigma(x) \sigma(y)}-\sum_{i=1}^{q} \sum_{x \in V} \widetilde{\alpha}_{i, x} \delta_{i \sigma(x)},
$$

where $J, \widetilde{\alpha}_{x}=\left(\widetilde{\alpha}_{1, x}, \ldots, \widetilde{\alpha}_{q, x}\right) \in \mathbb{R}^{q}$, and $\delta_{i j}$ is the Kronecker symbol.

If $J>0$ (resp. $J<0$ ), then the model is called a ferromagnetic (resp. antiferromagnetic) Potts model.

We define a finite-dimensional distribution by a probability measure $\mu$ in the volume $V_{n}$ as

$$
\mu_{n}\left(\sigma_{n}\right)=Z_{n}^{-1} \exp \left\{-\beta H_{n}\left(\sigma_{n}\right)+\sum_{x \in W_{n}} \widetilde{h}_{\sigma(x), x}\right\},
$$

where $\beta=1 / T, T>0$ is a temperature, $Z_{n}^{-1}$ is a normalization factor, $\left\{\widetilde{h}_{x}=\right.$ $\left.\left(\widetilde{h}_{1, x}, \ldots, \widetilde{h}_{q, x}\right) \in \mathbb{R}^{q}, x \in V\right\}$ is a collection of vectors, and

$$
H_{n}\left(\sigma_{n}\right)=-J \sum_{\langle x, y\rangle \in L_{n}} \delta_{\sigma(x) \sigma(y)}-\sum_{i=1}^{q} \sum_{x \in V_{n}} \widetilde{\alpha}_{i, x} \delta_{i \sigma(x)} .
$$

The distribution (2) is said to be compatible if the equality

$$
\sum_{\omega_{n} \in \Phi^{W_{n}}} \mu_{n}\left(\sigma_{n-1} \vee \omega_{n}\right)=\mu_{n-1}\left(\sigma_{n-1}\right)
$$

holds for all $n \geq 1$ and $\sigma_{n-1} \in \Phi^{V_{n-1}}$. Here, $\sigma_{n-1} \vee \omega_{n}$ is the union of configurations. In this case, there exists a unique measure $\mu$ on $\Phi^{V}$ such that for all $n$ and $\sigma_{n} \in \Phi^{V_{n}}$,

$$
\mu\left(\left\{\left.\sigma\right|_{V_{n}}=\sigma_{n}\right\}\right)=\mu_{n}\left(\sigma_{n}\right) .
$$

The measure is called the split Gibbs measure corresponding to Hamiltonian (1) and the vector-valued function $\widetilde{h}_{x}, x \in V$.

The following statement describes a condition for $\widetilde{h}_{x}$ and $\widetilde{\alpha}_{x}$ ensuring the consistency of $\mu_{n}\left(\sigma_{n}\right)$.

Theorem 2. Probability distributions $\mu_{n}\left(\sigma_{n}\right), n=1,2, \ldots$, in (2) are compatible iff for any $x \in V$ the following equation holds:

$$
h_{x}=\alpha_{x}+\sum_{y \in S(x)} F\left(h_{y}, \theta\right)
$$


where $\alpha_{x}=\left(\alpha_{1, x}, \alpha_{2, x}, \ldots, \alpha_{q-1, x}\right) \in \mathbb{R}^{q-1}, \alpha_{i, x}=\beta \widetilde{\alpha}_{i, x}-\beta \widetilde{\alpha}_{q, x} ; h_{x}=\left(h_{1, x}, \ldots\right.$, $\left.h_{q-1, x}\right), h_{i, x}=\widetilde{h}_{i, x}-\widetilde{h}_{q, x}+\left(\beta \widetilde{\alpha}_{i, x}-\beta \widetilde{\alpha}_{q, x}\right)$, and $F: h=\left(h_{1}, \ldots, h_{q-1}\right) \in \mathbb{R}^{q-1} \rightarrow$ $F(h, \theta)=\left(F_{1}, \ldots, F_{q-1}\right) \in \mathbb{R}^{q-1}$ is defined as

$$
F_{i}=\ln \left(\frac{(\theta-1) e^{h_{i}}+\sum_{j=1}^{q-1} e^{h_{j}}+1}{\theta+\sum_{j=1}^{q-1} e^{h_{j}}}\right),
$$

and $\theta=\exp (J \beta), S(x)$ is a set of direct successors of $x$.

P r o o f. The proof is similar to that of Theorem 5.1 in [4].

Let $G_{k} / G_{k}^{*}=\left\{H_{1}, \ldots, H_{r}\right\}$ be the quotient group for a normal divisor $G_{k}^{*}$ of index $r \geq 1$.

In this paper, we consider the case where $\alpha_{x}$ does not depend on $x$, i.e., $\alpha_{x} \equiv \alpha=\left(\alpha_{1}, \ldots, \alpha_{q-1}\right)$.

Definition 1. A collection of vectors $h=\left\{h_{x}, x \in G_{k}\right\}$ is said to be $G_{k}^{*}$ -periodic if $h_{y x}=h_{x}$ for all $x \in G_{k}, y \in G_{k}^{*} . A G_{k}-$ periodic collection is said to be translation-invariant. $S(x)$.

For $x \in G_{k}$, by $x_{\downarrow}$ we denote the unique point of the set $\left\{y \in G_{k}:\langle x, y\rangle\right\} \backslash$

Definition 2. A collection of vectors $h=\left\{h_{x}, x \in G_{k}\right\}$ is said to be $G_{k}^{*}$ -weakly periodic if $h_{x}=h_{i j}$ for $x \in H_{i}, x_{\downarrow} \in H_{j}, \forall x \in G_{k}$.

Definition 3. A measure $\mu$ is said to be $G_{k}^{*}$-periodic or weakly periodic if it corresponds to a $G_{k}^{*}$-periodic or a weakly periodic collection of vectors $h$, respectively.

\section{Weakly Periodic Measures}

The level of difficulty in describing weakly periodic Gibbs measures depends on the structure and index of the normal subgroup relative to which the periodicity condition is imposed. From [4], we know that in the group $G_{k}$, there is no normal subgroup of odd index different from one. Therefore, we consider normal subgroups of even indices. Here, we restrict ourself to the case of indices two.

Let $q \geq 3$ be arbitrary, i.e., $\sigma: V \rightarrow \Phi=\{1,2,3, \ldots, q\}$. We describe the $G_{k}^{*}$-weakly periodic Gibbs measures for a normal subgroup $G_{k}^{*}$ of index two. We note (see [4]) that any normal subgroup of index two of the group $G_{k}$ has the form

$$
H_{A}=\left\{x \in G_{k}: \sum_{i \in A} \omega_{x}\left(a_{i}\right)-\text { even }\right\},
$$


where $\emptyset \neq A \subseteq N_{k}=\{1,2, \ldots, k+1\}$, and $\omega_{x}\left(a_{i}\right)$ is the number of letters $a_{i}$ in the word $x \in G_{k}$. Let $A \subseteq N_{k}$ and $H_{A}$ be the corresponding normal subgroup of index two. We note that in the case $|A|=k+1$, i.e., in the case $A=N_{k}$, weak periodicity coincides with ordinary periodicity.

Consider $G_{k} / H_{A}=\left\{H_{A}, G_{k} \backslash H_{A}\right\}$ the quotient group.

For simplicity, we set $H_{0}=H_{A}, H_{1}=G_{k} \backslash H_{A}$. The $H_{A}$ are weakly periodic collections of vectors $h=\left\{h_{x} \in \mathbb{R}^{q-1}: x \in G_{k}\right\}$ have the form

$$
h_{x}= \begin{cases}h_{1}, & x_{\downarrow} \in H_{0}, x \in H_{0} \\ h_{2}, & x_{\downarrow} \in H_{0}, x \in H_{1} \\ h_{3}, & x_{\downarrow} \in H_{1}, x \in H_{0} \\ h_{4}, & x_{\downarrow} \in H_{1}, x \in H_{1} .\end{cases}
$$

Here $h_{i}=\left(h_{i 1}, h_{i 2}, \ldots, h_{i q-1}\right), i=1,2,3,4$. By (4), we then have

$$
\left\{\begin{array}{l}
h_{1}=(k-|A|) F\left(h_{1}, \theta\right)+|A| F\left(h_{2}, \theta\right) \\
h_{2}=(|A|-1) F\left(h_{3}, \theta\right)+(k+1-|A|) F\left(h_{4}, \theta\right) \\
h_{3}=(|A|-1) F\left(h_{2}, \theta\right)+(k+1-|A|) F\left(h_{1}, \theta\right) \\
h_{4}=(k-|A|) F\left(h_{4}, \theta\right)+|A| F\left(h_{3}, \theta\right) .
\end{array}\right.
$$

We introduce the notation $z_{i j}=\exp h_{i j}, \lambda_{j}=\exp \left(\alpha_{j}\right), i=1,2,3,4, j=$ $1,2, \ldots, q-1$. The last system of equations can then be rewritten as

$$
\left\{\begin{array}{l}
z_{1 j}=\lambda_{j}\left(\frac{(\theta-1) z_{1 j}+\sum_{i=1}^{q-1} z_{1 i}+1}{\sum_{i=1}^{q-1} z_{1 i}+\theta}\right)^{k-|A|}\left(\frac{(\theta-1) z_{2 j}+\sum_{i=1}^{q-1} z_{2 i}+1}{\sum_{i=1}^{q-1} z_{2 i}+\theta}\right)^{|A|} \\
z_{2 j}=\lambda_{j}\left(\frac{(\theta-1) z_{3 j}+\sum_{i=1}^{q-1} z_{3 i}+1}{\sum_{i=1}^{q-1} z_{3 i}+\theta}\right)^{|A|-1}\left(\frac{(\theta-1) z_{4 j}+\sum_{i=1}^{q-1} z_{4 i}+1}{\sum_{i=1}^{q-1} z_{4 i}+\theta}\right)^{k+1-|A|} \\
z_{3 j}=\lambda_{j}\left(\frac{(\theta-1) z_{2 j}+\sum_{i=1}^{q-1} z_{2 i}+1}{\sum_{i=1}^{q-1} z_{2 i}+\theta}\right)^{|A|-1}\left(\frac{(\theta-1) z_{1 j}+\sum_{i=1}^{q-1} z_{1 i}+1}{\sum_{i=1}^{q-1} z_{1 i}+\theta}\right)^{k+1-|A|} \\
z_{4 j}=\lambda_{j}\left(\frac{(\theta-1) z_{4 j}+\sum_{i=1}^{q-1} z_{4 i}+1}{\sum_{i=1}^{q-1} z_{4 i}+\theta}\right)^{k-|A|}\left(\frac{(\theta-1) z_{3 j}+\sum_{i=1}^{q-1} z_{3 i}+1}{\sum_{i=1}^{q-1} z_{3 i}+\theta}\right)^{|A|},
\end{array}\right.
$$

here $j=1,2,3, \ldots, q-1$.

We consider the map $K: \mathbb{R}^{4(q-1)} \rightarrow \mathbb{R}^{4(q-1)}$ defined as

$$
\left\{\begin{array}{l}
z_{1 j}^{\prime}=\lambda_{j}\left(\frac{(\theta-1) z_{1 j}+\sum_{i=1}^{q-1} z_{1 i}+1}{\sum_{i=1}^{q-1} z_{1 i}+\theta}\right)^{k-|A|}\left(\frac{(\theta-1) z_{2 j}+\sum_{i=1}^{q-1} z_{2 i}+1}{\sum_{i=1}^{q-1} z_{2 i}+\theta}\right)^{|A|} \\
z_{2 j}^{\prime}=\lambda_{j}\left(\frac{(\theta-1) z_{3 j}+\sum_{i=1}^{q-1} z_{3 i}+1}{\sum_{i=1}^{q-1} z_{3 i}+\theta}\right)^{|A|-1}\left(\frac{(\theta-1) z_{4 j}+\sum_{i=1}^{q-1} z_{4 i}+1}{\sum_{i=1}^{q-1} z_{4 i}+\theta}\right)^{k+1-|A|} \\
z_{3 j}^{\prime}=\lambda_{j}\left(\frac{(\theta-1) z_{2 j}+\sum_{i=1}^{q-1} z_{2 i}+1}{\sum_{i=1}^{q-1} z_{2 i}+\theta}\right)^{|A|-1}\left(\frac{(\theta-1) z_{1 j}+\sum_{i=1}^{q-1} z_{1 i}+1}{\sum_{i=1}^{q-1} z_{1 i}+\theta}\right)^{k+1-|A|} \\
z_{4 j}^{\prime}=\lambda_{j}\left(\frac{(\theta-1) z_{4 j}+\sum_{i=1}^{q-1} z_{4 i}+1}{\sum_{i=1}^{q-1} z_{4 i}+\theta}\right)^{k-|A|}\left(\frac{(\theta-1) z_{3 j}+\sum_{i=1}^{q-1} z_{3 i}+1}{\sum_{i=1}^{q-1} z_{3 i}+\theta}\right)^{|A|},
\end{array}\right.
$$

here $j=1,2,3, \ldots, q-1$. 
We introduce the notation

$$
\begin{gathered}
I_{m}=\left\{\left(z_{1}, z_{2}, \ldots, z_{q-1}\right) \in \mathbb{R}^{q-1}: z_{1}=z_{2}=\ldots=z_{m}, z_{m+1}=\ldots=z_{q-1}=1\right\}, \\
M_{m}=\left\{\left(z^{(1)}, z^{(2)}, z^{(3)}, z^{(4)}\right) \in \mathbb{R}^{4(q-1)}: z^{(i)} \in I_{m}, i=1,2,3,4\right\}
\end{gathered}
$$

here $m=1,2, \ldots, q-1$.

Lemma 1. 1) For any fixed $m \geq 1, \lambda>0$ and

$$
\lambda_{i}= \begin{cases}\lambda & \text { if } 1 \leq i \leq m \\ 1 & \text { if } m<i \leq q-1\end{cases}
$$

the set $M_{m}$ is an invariant set with respect to the map $K$, i.e., $K\left(M_{m}\right) \subset M_{m}$. 2) For $\alpha=0$, the sets $M_{m}$ are invariant with respect to the map $K$ for all $m=1,2, \ldots, q-1$.

Case $\alpha \neq 0$. Let us consider the case $\alpha \neq 0$ and $\lambda_{i}$ given by (10). For $\mathbf{z} \in M_{m}$, we denote $z_{i}=z_{i j}, i=1,2,3,4 ; j=1,2, \ldots, m$.. Then on the invariant set $M_{m}$ the system of equations (6) has the form

$$
\left\{\begin{aligned}
z_{1} & =\lambda\left(\frac{(\theta+m-1) z_{1}+q-m}{m z_{1}+\theta+q-m-1}\right)^{k-|A|}\left(\frac{(\theta+m-1) z_{2}+q-m}{m z_{2}+\theta+q-m-1}\right)^{|A|} \\
z_{2} & =\lambda\left(\frac{(\theta+m-1) z_{3}+q-m}{m z_{3}+\theta+q-m-1}\right)^{|A|-1}\left(\frac{(\theta+m-1) z_{4}+q-m}{m z_{4}+\theta+q-m-1}\right)^{k+1-|A|} \\
z_{3} & =\lambda\left(\frac{(\theta+m-1) z_{2}+q-m}{m z_{2}+\theta+q-m-1}\right)^{|A|-1}\left(\frac{(\theta+m-1) z_{1}+q-m}{m z_{1}+\theta+q-m-1}\right)^{k+1-|A|} \\
z_{4} & =\lambda\left(\frac{(\theta+m-1) z_{4}+q-m}{m z_{4}+\theta+q-m-1}\right)^{k-|A|}\left(\frac{(\theta+m-1) z_{3}+q-m}{m z_{3}+\theta+q-m-1}\right)^{|A|} .
\end{aligned}\right.
$$

We introduce the notation

$$
f_{m}(z)=\frac{(\theta+m-1) z+q-m}{m z+\theta+q-m-1} .
$$

It is easy to prove the following

Lemma 2. The function $f_{m}(z)$ is strictly decreasing for $0<\theta<1,1 \leq m \leq$ $q-1$ and it is strictly increasing for $1<\theta$.

Proposition 1. Let $\boldsymbol{z}=\left(z_{1}, z_{2}, z_{3}, z_{4}\right)$ be a solution of the system of equations (11). If $z_{i}=z_{j}$ for some $i \neq j$, then $z_{1}=z_{2}=z_{3}=z_{4}$.

We consider an antiferromagnetic Potts model (i.e., $0<\theta<1$ ).

Let $|A|=k$. Then the system of equations (11) has the form

$$
\left\{\begin{array}{l}
z_{1}=\lambda\left(f_{m}\left(z_{2}\right)\right)^{k} \\
z_{2}=\lambda\left(f_{m}\left(z_{3}\right)\right)^{k-1}\left(f_{m}\left(z_{4}\right)\right) \\
z_{3}=\lambda\left(f_{m}\left(z_{2}\right)\right)^{k-1}\left(f_{m}\left(z_{1}\right)\right) \\
z_{4}=\lambda\left(f_{m}\left(z_{3}\right)\right)^{k}
\end{array}\right.
$$


The solving of the system (12) can be reduced to the analyzing of the following system of equations:

$$
\left\{\begin{array}{l}
z_{2}=\lambda\left(f_{m}\left(z_{3}\right)\right)^{k-1} \cdot f_{m}\left(\lambda\left(f_{m}\left(z_{3}\right)\right)^{k}\right) \\
z_{3}=\lambda\left(f_{m}\left(z_{2}\right)\right)^{k-1} \cdot f_{m}\left(\lambda\left(f_{m}\left(z_{2}\right)\right)^{k}\right) .
\end{array}\right.
$$

Introduce the notation

$$
\psi(z)=\lambda\left(f_{m}(z)\right)^{k-1} \cdot f_{m}\left(\lambda\left(f_{m}(z)\right)^{k}\right) .
$$

Then we reduce the system of equations (13) to the form

$$
\left\{\begin{array}{l}
z_{2}=\psi\left(z_{3}\right) \\
z_{3}=\psi\left(z_{2}\right)
\end{array}\right.
$$

The number of solutions of the system (15) coincides with the number of solutions of the equation $\psi(\psi(z))=z$.

Lemma 3. Let $\gamma:[0,1] \rightarrow[0,1]$ be a continuous function with a fixed point $\xi \in(0,1)$. Assuming that the function $\gamma$ is differentiable at $\xi \in(0,1)$ and that $\gamma^{\prime}(\xi)<-1$, we have the values $x_{0}, x_{1}$ such that the inequalities $0 \leq x_{0}<\xi<$ $x_{1} \leq 1$ hold and $\gamma\left(x_{0}\right)=x_{1}, \gamma\left(x_{1}\right)=x_{0}$ (see [16], $p$. 70).

It is known (see [4], p. 109) that for antiferromagnetic case we have a unique translation-invariant Gibbs measure corresponding to the unique solution of the equation $z=\lambda f_{m}^{k}(z)$. We let $z_{*}$ denote this solution.

Proposition 2. For $k \geq 6$ and $\lambda \in\left(\lambda_{c_{1}}, \lambda_{c_{2}}\right)$, the system of equations (15) has three solutions $\left(z_{*}, z_{*}\right),\left(z_{2}^{*}, z_{3}^{*}\right),\left(z_{3}^{*}, z_{2}^{*}\right)$, where $\lambda_{c_{i}}=b_{i}^{k}, i=1,2$, and

$$
\begin{aligned}
& b_{1}=\frac{\left(k-1-\sqrt{k^{2}-6 k+1}\right)(1-\theta)(\theta+q-1) z_{*}^{\frac{k-1}{k}}}{2\left(m z_{*}+\theta+q-m-1\right)^{2}}, \\
& b_{2}=\frac{\left(k-1+\sqrt{k^{2}-6 k+1}\right)(1-\theta)(\theta+q-1) z_{*}^{\frac{k-1}{k}}}{2\left(m z_{*}+\theta+q-m-1\right)^{2}} .
\end{aligned}
$$

We have thus the following theorem.

Theorem 3. Let $|A|=k, k \geq 6$, and $\lambda \in\left(\lambda_{c_{1}}, \lambda_{c_{2}}\right)$. Then for the antiferromagnetic Potts model with special external field (given by (10)) there are at least two $H_{A}$ - weakly periodic (non-periodic) Gibbs measures, where $\lambda_{c_{i}}=b_{i}^{k}, i=1,2$. 
Case $\alpha=0$. In this case, the system of equations (6) on the invariant set $M_{m}, m=1,2, \ldots, q-1$ can be reduced to the following system of equations:

$$
\left\{\begin{aligned}
z_{1} & =\left(\frac{(\theta+m-1) z_{1}+q-m}{m z_{1}+\theta+q-m-1}\right)^{k-|A|}\left(\frac{(\theta+m-1) z_{2}+q-m}{m z_{2}+\theta+q-m-1}\right)^{|A|} \\
z_{2} & =\left(\frac{(\theta+m-1) z_{3}+q-m}{m z_{3}+\theta+q-m-1}\right)^{|A|-1}\left(\frac{(\theta+m-1) z_{4}+q-m}{m z_{4}+\theta+q-m-1}\right)^{k+1-|A|} \\
z_{3} & =\left(\frac{(\theta+m-1) z_{2}+q-m}{m z_{2}+\theta+q-m-1}\right)^{|A|-1}\left(\frac{(\theta+m-1) z_{1}+q-m}{m z_{1}+\theta+q-m-1}\right)^{k+1-|A|} \\
z_{4} & =\left(\frac{(\theta+m-1) z_{4}+q-m}{m z_{4}+\theta+q-m-1}\right)^{k-|A|}\left(\frac{(\theta+m-1) z_{3}+q-m}{m z_{3}+\theta+q-m-1}\right)^{|A|} .
\end{aligned}\right.
$$

The following proposition is similar to Proposition 1.

Proposition 3. Let $m \in\{1,2, \ldots, q-1\}$ be fixed and $\boldsymbol{z}=\left(z_{1}, z_{2}, z_{3}, z_{4}\right)$ be a solution of the system of equations (17). If $z_{i}=z_{j}$ for some $i \neq j$, then $z_{1}=z_{2}=z_{3}=z_{4}$.

Theorem 4. Let $|A|=k$ and $k \geq 6$. If one of the following conditions is satisfied:

1) $\frac{4 k}{k+1+\sqrt{k^{2}-6 k+1}} \leq q<\frac{4 k}{k+1-\sqrt{k^{2}-6 k+1}} \quad 0<\theta<\theta_{2}$,

2) $q \leq \frac{4 k}{k+1+\sqrt{k^{2}-6 k+1}} \quad \theta_{1}<\theta<\theta_{2}$,

then there are at least $2^{q}-2$ weakly periodic (non-periodic) Gibbs measures, where

$$
\theta_{1}=\frac{4 k-k q-q-q \sqrt{k^{2}-6 k+1}}{4 k}, \quad \theta_{2}=\frac{4 k-k q-q+q \sqrt{k^{2}-6 k+1}}{4 k} .
$$

$\mathrm{R}$ e $\mathrm{m}$ a $\mathrm{rk}$ 1. The new Gibbs measures described in Theorem 3 and Theorem 4 allow us to describe a continuous set of non-periodic Gibbs measures different from the previously known ones.

$\mathrm{R}$ e $\mathrm{m}$ a r k 2. If instead of (9) we consider $M_{q-1}$, then Theorem 4 coincides with Theorem 3 from [13].

\section{Proofs}

$\mathrm{P}$ r o o f of Lemma 1. 1) For the fixed $m, \lambda_{i}$ given by (10) and $\mathbf{z}=$ $\left(z^{(1)}, z^{(2)}, z^{(3)}, z^{(4)}\right) \in M_{m}$, it is clear that $z^{(i)} \in I_{m}, i=1,2,3,4$. From (8), we have that $z^{(i)}=\left(z_{i}, \ldots, z_{i}, 1, \ldots, 1\right)$, where $z_{i} \neq 1, i=1,2,3,4$. Using $(7)$, we obtain 


$$
\left\{\begin{aligned}
z_{1 j}^{\prime} & =\left(\frac{\theta+q-2+z_{1}}{\theta+q-2+z_{1}}\right)^{k-|A|}\left(\frac{\theta+q-2+z_{2}}{\theta+q-2+z_{2}}\right)^{|A|}=1, j=m, m+1, \ldots q-1 \\
z_{2 j}^{\prime} & =\left(\frac{\theta+q-2+z_{3}}{\theta+q-2+z_{3}}\right)^{|A|-1}\left(\frac{\theta+q-2+z_{4}}{\theta+q-2+z_{4}}\right)^{k+1-|A|}=1, j=m, m+1, \ldots q-1 \\
z_{3 j}^{\prime} & =\left(\frac{\theta+q-2+z_{2}}{\theta+q-2+z_{2}}\right)^{|A|-1}\left(\frac{\theta+q-2+z_{4}}{\theta+q-2+z_{4}}\right)^{k+1-|A|}=1, j=m, m+1, \ldots q-1 \\
z_{4 j}^{\prime} & =\left(\frac{\theta+q-2+z_{4}}{\theta+q-2+z_{4}}\right)^{k-|A|}\left(\frac{\theta+q-2+z_{3}}{\theta+q-2+z_{3}}\right)^{|A|}=1, j=m, m+1, \ldots q-1
\end{aligned}\right.
$$

Consequently, $K(\mathbf{z}) \in M_{m}$.

The second part of the lemma is proved in a similar way.

P r o of of Proposition 1

From the system of equations (11), we obtain

$$
\begin{aligned}
& \frac{z_{1}}{z_{2}}=\left(\frac{f_{m}\left(z_{1}\right)}{f_{m}\left(z_{4}\right)}\right)^{k-|A|}\left(\frac{f_{m}\left(z_{2}\right)}{f_{m}\left(z_{3}\right)}\right)^{|A|-1}\left(\frac{f_{m}\left(z_{2}\right)}{f_{m}\left(z_{4}\right)}\right), \\
& \frac{z_{1}}{z_{3}}=\left(\frac{f_{m}\left(z_{2}\right)}{f_{m}\left(z_{1}\right)}\right), \\
& \frac{z_{1}}{z_{4}}=\left(\frac{f_{m}\left(z_{1}\right)}{f_{m}\left(z_{4}\right)}\right)^{k-|A|}\left(\frac{f_{m}\left(z_{2}\right)}{f_{m}\left(z_{3}\right)}\right)^{|A|}, \\
& \frac{z_{2}}{z_{3}}=\left(\frac{f_{m}\left(z_{3}\right)}{f_{m}\left(z_{2}\right)}\right)^{|A|-1}\left(\frac{f_{m}\left(z_{4}\right)}{f_{m}\left(z_{1}\right)}\right)^{k-|A|+1}, \\
& \frac{z_{2}}{z_{4}}=\left(\frac{f_{m}\left(z_{4}\right)}{f_{m}\left(z_{3}\right)}\right) \text {, } \\
& \frac{z_{3}}{z_{4}}=\left(\frac{f_{m}\left(z_{1}\right)}{f_{m}\left(z_{4}\right)}\right)^{k-|A|}\left(\frac{f_{m}\left(z_{2}\right)}{f_{m}\left(z_{3}\right)}\right)^{|A|-1}\left(\frac{f_{m}\left(z_{1}\right)}{f_{m}\left(z_{3}\right)}\right) .
\end{aligned}
$$

Let $\mathbf{z}=\left\{z_{1}, z_{2}, z_{3}, z_{4}\right\}$ be a solution of the system of equations (11) and $z_{1}=z_{2}$. Then from the strict monotonicity of $f_{m}(z)$ and from the equality $\left(a_{2}\right)$, we obtain that $z_{1}=z_{2}=z_{3}$. In this case, from $\left(a_{4}\right)$ we obtain $z_{1}=z_{4}$, consequently, $z_{1}=z_{2}=z_{3}=z_{4}$.

Let $z_{1}=z_{3}$. Then from the strict monotonicity of $f_{m}(z)$ and from the equality $\left(a_{2}\right)$, we obtain that $z_{1}=z_{2}=z_{3}$. In this case, from $\left(a_{4}\right)$ we obtain $z_{1}=z_{4}$, consequently, $z_{1}=z_{2}=z_{3}=z_{4}$.

Let $z_{1}=z_{4}$. Then from the strict monotonicity of $f_{m}(z)$ and from the equality $\left(a_{3}\right)$, we obtain that $z_{2}=z_{3}$. Then from $\left(a_{5}\right)$, we obtain the inequality

$$
z_{2} f_{m}\left(z_{2}\right)=z_{4} f_{m}\left(z_{4}\right)
$$


We consider the function

$$
\phi(z)=z f_{m}(z)=z \frac{(\theta+m-1) z+q-m-1}{m z+\theta+q-m-1}
$$

and calculate its derivative

$$
\phi^{\prime}(z)=\frac{(\theta-1+m) m z^{2}+2(\theta-1+m)(\theta+q-m-1) z+(q-m)(\theta+q-m-1)}{(m z+\theta+q-m-1)^{2}} .
$$

From $\theta>0, \quad z>0, \quad 1 \leq m \leq q-1$ and $q \geq 2$, we obtain that $\phi(z)$ is a strictly increasing function. Consequently, (19) is true only when $z_{2}=z_{4}$.

The other cases are proved in a similar way.

P r o o f of Proposition 2. For (14), it is easy to see that

1) $\psi\left(z_{*}\right)=z_{*}$,

2) the function $\psi(z)$ is defined on $\mathbb{R}_{+}$,

3) $\psi(z)$ is bounded and differentiable at $z_{*}$.

Then, by Lemma 1 , for $\psi^{\prime}\left(z_{*}\right)<-1$, equation (15) has three solutions of the form $\left(z_{*}, z_{*}\right),\left(z_{2}^{*}, z_{3}^{*}\right),\left(z_{3}^{*}, z_{2}^{*}\right)$. The inequality $\psi^{\prime}\left(z_{*}\right)<-1$ is equivalent to the inequality

$$
b^{2}+(k-1) \frac{(\theta-1)(\theta+q-1) z_{*}^{\frac{k-1}{k}}}{\left(m z_{*}+\theta+q-m-1\right)^{2}} b+k \frac{(1-\theta)^{2}(\theta+q-1)^{2} z_{*}^{2 \frac{k-1}{k}}}{\left(m z_{*}+\theta+q-m-1\right)^{4}}<0,
$$

where $b=\sqrt[k]{\lambda}$. Consequently,

$$
\left(b-b_{1}\right)\left(b-b_{2}\right)<0,
$$

where $b_{1}, b_{2}$ are defined in (16). Proposition 2 is proved.

P r o of of Theorem 4 .

Consider the case $0<\theta<1$ and $|A|=k$. Then the system of equations (17) has the form

$$
\left\{\begin{array}{l}
z_{1}=\left(f_{m}\left(z_{2}\right)\right)^{k} \\
z_{2}=\left(f_{m}\left(z_{3}\right)\right)^{k-1}\left(f_{m}\left(z_{4}\right)\right) \\
z_{3}=\left(f_{m}\left(z_{2}\right)\right)^{k-1}\left(f_{m}\left(z_{1}\right)\right) \\
z_{4}=\left(f_{m}\left(z_{3}\right)\right)^{k} .
\end{array}\right.
$$

Reduce (21) to the system

$$
\left\{\begin{array}{l}
z_{2}=\left(f_{m}\left(z_{3}\right)\right)^{k-1} \cdot f_{m}\left(\left(f_{m}\left(z_{3}\right)\right)^{k}\right) \\
z_{3}=\left(f_{m}\left(z_{2}\right)\right)^{k-1} \cdot f_{m}\left(\left(f_{m}\left(z_{2}\right)\right)^{k}\right)
\end{array}\right.
$$

After introducing the notation

$$
\varphi(z)=\left(f_{m}(z)\right)^{k-1} \cdot f_{m}\left(\left(f_{m}(z)\right)^{k}\right),
$$


the system of equations (22) can by written as follows:

$$
\left\{\begin{array}{l}
z_{2}=\varphi\left(z_{3}\right) \\
z_{3}=\varphi\left(z_{2}\right)
\end{array}\right.
$$

Note that the following hold:

1) $\varphi(1)=1$,

2) the function $\varphi(z)$ is defined on $\mathbb{R}_{+}$,

3) $\varphi(z)$ is bounded and differentiable at $z=1$.

Therefore, by Lemma 3 , for $\varphi^{\prime}(1)<-1$, the system of equations (24) has three solutions of the form $(1,1),\left(z_{2}^{*}, z_{3}^{*}\right),\left(z_{3}^{*}, z_{2}^{*}\right)$. The inequality $\varphi^{\prime}(1)<-1$ is equivalent to the inequality

$$
k \frac{(\theta-1)^{2}}{(\theta+q-1)^{2}}+(k-1) \frac{(\theta-1)}{(\theta+q-1)}+1<0 .
$$

Consequently ,

$$
2 k\left(\theta-\theta_{1}\right)\left(\theta-\theta_{2}\right)<0,
$$

where $\theta_{1}, \theta_{2}$ are defined in $(12)$.

It is clear that if $k<5$, then $\theta_{1}, \theta_{2}$ are complex numbers, if $k=5$, then $\theta_{1}=\theta_{2}$ and inequality (25) has no solution.

We now assume that $k \geq 6$. We suppose that $\theta_{1}, \theta_{2}$ are simultaneously negative. Then (25) has no solution. If $\theta_{1} \leq 0,0<\theta_{2}<1$, i.e.,

$$
\frac{4 k}{k+1+\sqrt{k^{2}-6 k+1}} \leq q<\frac{4 k}{k+1-\sqrt{k^{2}-6 k+1}},
$$

then inequality (25) has the solution $\theta \in\left(0, \theta_{2}\right)$, which proves item 1 in Theorem 3 . Let $0 \leq \theta_{1}<1,0<\theta_{2}<1$, then the inequality

$$
q \leq \frac{4 k}{k+1+\sqrt{k^{2}-6 k+1}}
$$

is satisfied.

Inequality (25) has the solution $\theta_{1}<\theta<\theta_{2}$. It is easy to see that $\theta_{1}, \theta_{2}$ are less or equal to 1 . Thus, by Theorem 2 , for every $m$ and under conditions of Theorem 4, we obtain two weakly periodic (non-periodic) Gibbs measures. From (8), we note that $m$ is the number of coordinates other than 1 of the vectors from $\mathbb{R}^{q-1}$. It is obvious that the number of these vectors is equal to $\sum_{m=1}^{q-1} C_{q-1}^{m}=2^{q-1}-1$. Consequently, when the conditions of Theorem 4 are satisfied, we obtain the $2\left(2^{q-1}-1\right)=2^{q}-2$ weakly periodic (non-periodic) Gibbs measures. Theorem 4 is proved.

Acknowledgment. The author thanks Professor U.A. Rozikov for useful discussions. 


\section{References}

[1] H.O. Georgii, Gibbs Measures and Phase Transitions. de Gruyter, Berlin, 1988.

[2] C.J. Preston, Gibbs States on Countable Sets. Cambridge Tracts Math., 68, Cambridge Univ. Press, Cambridge, 1974.

[3] Ya.G. Sinai, Theory of Phase Transitions: Rigorous Results. Nauka, Moscow, 1980. (Russian). (Engl. transl.: Intl. Series Nat. Philos., Vol. 108), Pergamon Press, Oxford, 1982.

[4] U.A. Rozikov, Gibbs measures on Cayley trees. World scientific, 2013.

[5] N.N. Ganikhodzhaev, Pure Phases of the Ferromagnetic Potts Model with Three States on a Second-Order Bethe Lattice. - Theor. Math. Phys. 85 (1990), No. 2, $1125-1134$.

[6] N.N. Ganikhodzhaev, Pure Phases of the Ferromagnetic Potts Model on the Bethe Lattice. - Dokl. AN RUz 67 (1992), 4-7.

[7] N.N. Ganikhodzhaev and U.A. Rozikov, Description of Periodic Extreme Gibbs Measures of Some Lattice Models on the Cayley Tree. - Theor. Math. Phys. 111 (1992), No. 1, 480-486.

[8] N.N. Ganikhodzhaev and U.A. Rozikov, The Potts Model with Countable Set of Spin Values on a Cayley Tree. — Lett. Math. Phys. 75 (2006), No. 2, 99-109.

[9] C. Külske, U.A. Rozikov, and R.M. Khakimov, Description of Translation-Invariant Splitting Gibbs Measures for the Potts Model on a Cayley Tree. - J. Stat. Phys. 156 (2014), No. 1, 189-200.

[10] U.A. Rozikov and N.N. Ganikhodzhaev, On Weak Periodic Gibbs Measures of Ising Model on Cayley Trees. — Theor. Math. Phys. 156 (2008), No. 2, 1218-1227.

[11] U.A. Rozikov and M.M. Rakhmatullaev, Weakly Periodic Ground States and Gibbs Measures for the Ising Model with Competing Interactions on the Cayley Tree. Theor. Math. Phys. 160 (2009), No. 3, 1292-1300.

[12] M.M. Rakhmatullaev, Weakly Periodic Gibbs Measures and Ground States for the Potts Model with Competing Inter-Actions on the Cayley Tree. - Theor. Math. Phys. 176 (2013), No. 3, 1236-1251.

[13] M.M. Rakhmatullaev, The Existence of Weakly Periodic Gibbs Measures for the Potts Model on a Cayley Tree. - Theor. Math. Phys. 180 (2014), No. 3, 10191029.

[14] N.N. Ganikhodjaev and U.A. Rozikov, Group Representation of the Cayley Forest and Some of its Applications. - Izvestiya: Math. 67 (2003), No. 1, 17-27.

[15] N.N. Ganikhodjaev, Group Representations and Automorphisms of a Cayley Tree. - Dokl. AN RUz 4 (1994), 3-5.

[16] H. Kesten, Quadratic Transformations: a Model for Population Growth. I. - Adv. Appl. Probab. 2 (1970), 1-82. 